ARTICLE

https://doi.org/10.1038/s41467-019-08434-4

\title{
Stable radical anions generated from a porous perylenediimide metal-organic framework for boosting near-infrared photothermal conversion
}

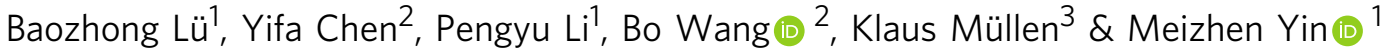

Radical anions of electron-deficient systems are widely used, but are easily reoxidized upon exposure to air. Therefore, the stabilization of radical anions under ambient conditions is of great significance, but still remains a scientific challenge. Herein, perylenediimide is employed to prepare a crystalline metal-organic framework for stabilizing radical anions without extensive chemical modification. The porous, three-dimensional framework of perylenediimide can trap electron donors such as amine vapors and produce radical anions in-situ through photo-induced electron transfer. The radical anions are protected against quenching by shielding effect in air and remain unobstructed in air for at least a month. Because of the high yield and stability of the radical anions, which are the basis for nearinfrared photothermal conversion, the framework shows high near-infrared photothermal conversion efficiency $(\eta=52.3 \%)$. The work provides an efficient and simple method towards ambient stable radical anions and affords a promising material for photothermal therapy.

\footnotetext{
${ }^{1}$ State Key Laboratory of Chemical Resource Engineering, Beijing Advanced Innovation Center for Soft Matter Science and Engineering, Beijing Laboratory of Biomedical Materials, Beijing University of Chemical Technology, 100029 Beijing, P. R. China. ${ }^{2}$ Key Laboratory of Cluster Science, Ministry of Education, School of Chemistry and Chemical Engineering, Beijing Institute of Technology, 100081 Beijing, P. R. China. ${ }^{3}$ Max Planck Institute for Polymer Research, Institute of Physical Chemistry, Johannes Gutenberg University Mainz, Duesbergweg 10-14, 55128 Mainz, Germany. These authors contributed equally: Baozhong Lü, Yifa Chen. Correspondence and requests for materials should be addressed to M.Y. (email: yinmz@mail.buct.edu.cn)
} 
$\mathrm{N}$ ear-infrared (NIR) photothermal materials convert absorbed NIR light into thermal energy by inhibiting the radiative relaxation of materials ${ }^{1,2}$. Because of the deep penetration ability and dark field viewing properties of NIR light, the NIR photothermal effect has been of interest in applications such as NIR photothermal ablation ${ }^{3}$, NIR laser-assisted photothermal therapy ${ }^{4}$, and night vision sensors $^{5}$. Conventional organic NIR-absorbing molecules may not only require complex synthesis procedures but also easily suffer from photobleaching under light irradiation. These drawbacks lead to high costs and a risk of performance decay in photothermal processes. Thus, it is essential to devise an organic photothermal agent with stable photothermal capacity.

Perylenediimides (PDIs) are an outstanding class of organic dye molecules that have been utilized for nearly a century ${ }^{6-8}$. Owing to the ease of reduction into delocalized radical anions $(\mathrm{RAs})^{9}$, these versatile organic molecules are of interest in photocatalysis $^{10}$, n-channel transistor fabrication ${ }^{11}$, and photoconduction $^{12}$. PDI RAs $\left(\mathrm{PDI}^{\bullet^{-}}\right)$display a typical red-shift absorption towards the NIR region. Such useful NIR absorbers play an important role as photothermal agents for photothermal therapy. Photothermal therapy has significant advantages over chemotherapy and radiotherapy due to its superior local efficacy and fewer systemic side effects ${ }^{2-4}$. PDI ${ }^{\bullet-}$ have already been used to regulate the balance of bacterial flora ${ }^{13-15}$. However, these applications, especially NIR photothermal conversion, are adversely affected by the instability of the RAs under ambient conditions ${ }^{16-26}$. The formation and use of ambient stable PDI ${ }^{\bullet-}$ thus remain of prime importance. Many efforts have been devoted to improving the stability of RAs, such as covalently modifying PDI cores and incorporating RAs into films or supramolecular assemblies ${ }^{13,20-23}$. These efforts are often demanding from both a synthesis and a processing point of view. Except for an imidazolium-modified zwitterionic PDI, the RAs are rapidly reoxidized upon exposure to $\mathrm{air}^{20}$. Functionalized PDI materials without extensive chemical modification, but yielding stable RAs under ambient conditions, would be highly desirable.

Metal-organic frameworks (MOFs) are a class of robust, porous, crystalline hybrid materials that have received much attention due to their myriad of properties and simple synthesis ${ }^{27-31}$. It is indeed possible to obtain rationally designed functional MOFs by selecting various metal ions and organic ligands or by adjusting the synthesis conditions. Among other functions, threedimensional (3D) porous MOFs can serve as stable cages $^{32-34}$. However, the known MOFs do not on their own produce RAs because of the lack of strongly electron-deficient aromatic ligands. We reasoned that cages composed of PDI ligands could produce RAs in situ with improved stability due to a pronounced shielding effect. PDI-based 3D MOFs have not been reported because (1) PDIs with coordinating carboxyl or pyridyl functions exhibit poor solubility in common solvents and (2) PDIs tend to form onedimensional or two-dimensional assemblies with close $\pi-\pi$ stacking, which inhibits porosity ${ }^{7,35-38}$.

To elucidate this concept, we report a PDI-based 3D MOF, Zr-PDI, composed of a $N, N^{\prime}$-di-(4-benzoic acid)-1,2,6,7-tetrachloroperylene-3,4,9,10-tetracarboxylic acid diimide (P-2COOH) ligand and $\mathrm{Zr}_{6}\left(\mu_{3}-\mathrm{O}\right)_{4}\left(\mu_{3}-\mathrm{OH}\right)_{4}$ clusters (Fig. 1). The non-planar structure of $\mathrm{P}-2 \mathrm{COOH}$ enhances its solubility in polar solvents, thus allowing the successful solvothermal synthesis of Zr-PDI. $\mathrm{Zr}^{4+}$ is chosen because the reported $\mathrm{Zr}$-based MOFs exhibit remarkable stability and high porosity ${ }^{29}$. Zr-PDI forms a fascinating $3 \mathrm{D}$ porous network and can serve as cage to trap electron donors such as organic amines to furnish RAs (Zr-PDI ${ }^{\bullet-}$ ) in situ by photo-induced electron transfer (PET). Being confined in a framework, $\mathrm{Zr}$-PDI ${ }^{-}$has superior stability in air and remains intact for at least a month. To the best of our knowledge,
$\mathrm{Zr}-\mathrm{PDI}^{\bullet-}$ is the first example of isolated RAs which are stable at ambient conditions without modification. A similar stability cannot be obtained by using the ligand $\mathrm{P}-2 \mathrm{COOH}^{\bullet-}$ under the same conditions. Zr-PDI ${ }^{\bullet}$, with NIR absorbance, in addition to high yield and stability, shows an exceptionally high NIR photothermal conversion efficiency $(\eta=52.3 \%)$ and good recyclability. In comparison, $\mathrm{P}-2 \mathrm{COOH}^{\bullet-}$ shows poor NIR photothermal conversion efficiency and recyclability.

\section{Results and discussion}

Framework formation and structural characterization. The solvothermal reaction of P-2COOH (Supplementary Figs 1 and 2) and $\mathrm{ZrCl}_{4}$ in dimethyl formamide (DMF) in the presence of acetic acid as a modulator (a coordination modulator controls coordination kinetics and the resultant crystal size) produced Zr-PDI in a yield of $58 \%$. Single-crystal analysis revealed that Zr-PDI crystallized in the tetragonal space group I41/a (Supplementary Table 1). In the $\mathrm{Zr}_{6}\left(\mu_{3}-\mathrm{O}\right)_{4}\left(\mu_{3}-\mathrm{OH}\right)_{4}$ octahedral cluster formed, the six vertices of the octahedron are occupied by $\mathrm{Zr}(\mathrm{IV})$ centers and the eight triangular faces are alternatively capped by four $\mu_{3}-\mathrm{OH}$ and four $\mu_{3}-\mathrm{O}$ groups, as shown in Fig. 1a. The $\mathrm{Zr}_{6}$ $\left(\mu_{3}-\mathrm{O}\right)_{4}\left(\mu_{3}-\mathrm{OH}\right)_{4}$ cluster is fully coordinated by 12 carboxylate units (i.e. $8 \mathrm{P}-2 \mathrm{COOH}$ ligands and 4 acetic acid molecules) (Fig. 1b). From a topological point of view, a 3D framework originates from a highly symmetric $\mathrm{Zr}_{6}\left(\mu_{3}-\mathrm{O}\right)_{4}\left(\mu_{3}-\mathrm{OH}\right)_{4}-\left(\mathrm{CO}_{2}\right)_{12}$ secondary building unit and organic ligands (Fig. 1c). Owing to the electrostatic repulsion and steric hindrance between the chloro substituents, the central six-membered ring of $\mathrm{P}-2 \mathrm{COOH}$ is highly twisted with a dihedral angle of $38.3^{\circ}$ (Supplementary Fig. 3) associated with the bay carbon atoms C11-C12-C20-C21. This twist also induces the axial chirality of PDIs, which can be observed in the crystalline state (Fig. $1 \mathrm{~b})^{39}$. Figure $1 \mathrm{~b}$ shows two $\mathrm{P}-2 \mathrm{COOH}$ ligands with opposite chirality, which serve as a bridge connecting two $\mathrm{Zr}_{6}\left(\mu_{3}-\mathrm{O}\right)_{4}\left(\mu_{3}-\mathrm{OH}\right)_{4}$ clusters.

MOFs incorporating extended linkers such as $\mathrm{P}-2 \mathrm{COOH}$ (length $\sim 24 \AA$ ) are often unstable and undergo pore collapse upon solvent removal. Figure 1d displays a huge distorted adamantanoid cage in Zr-PDI. While minimizing the large cavities in the adamantanoid cages, a normal five-fold nonconnected interpenetration is formed, as illustrated in Supplementary Fig. 4. Upon inspection of the powder X-ray diffraction (PXRD) patterns, it is clear that Zr-PDI is highly crystalline, consistent with the simulated pattern (Supplementary Fig. 5). Scanning electron microscopy (SEM) images reveal that the bulk microcrystalline powder consists of cubic crystallites $\sim 2 \mu \mathrm{m}$ in length and with a smooth surface (Supplementary Figs 6 and 7). Stability tests were performed by incubating the as-synthesized $\mathrm{Zr}$-PDI under various conditions, and the results are shown in Fig. 1e. Identical PXRD patterns were obtained before and after incubation, confirming the stability of the framework. $\mathrm{N}_{2}$ sorption analysis of Zr-PDI gave a Brunauer-Emmett-Teller (BET) surface area of $1330 \mathrm{~m}^{2} / \mathrm{g}$ and a total pore volume of $0.71 \mathrm{~cm}^{3} / \mathrm{g}$ by a volumetric method, comparable to those of $\mathrm{UiO}$ 66 (ref. ${ }^{29}$ ). Type I isotherms are observed for Zr-PDI (Fig. 1f), indicating dominating microporous adsorption. Indeed, Zr-PDI exhibits mainly two types of pores with sizes of $\sim 12$ and $\sim 15 \AA$.

Optical properties and formation of RAs. The arrangement of $\mathrm{P}-2 \mathrm{COOH}$ molecules within the framework makes it a remarkable model to study the optical properties of Zr-PDI (Supplementary Figs 8-12). In the UV-Vis-NIR absorption spectrum in Fig. 2a, Zr-PDI exhibits mainly three PDI absorptions in the $450-560 \mathrm{~nm}$ range, which are similar to the absorptions of alkyl-substituted PDI thin film ${ }^{40}$. Zr-PDI also gives a red-shift of the broad absorption in the visible region from that of the 
a

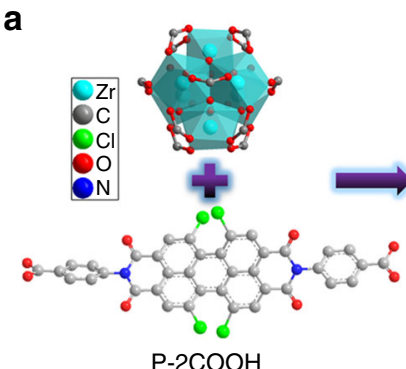

d

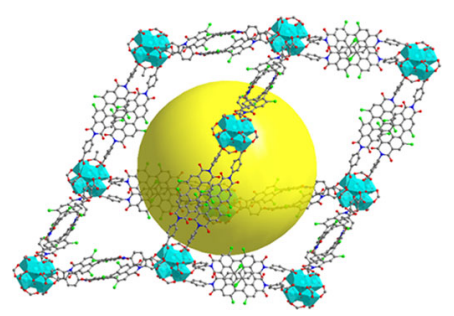

b

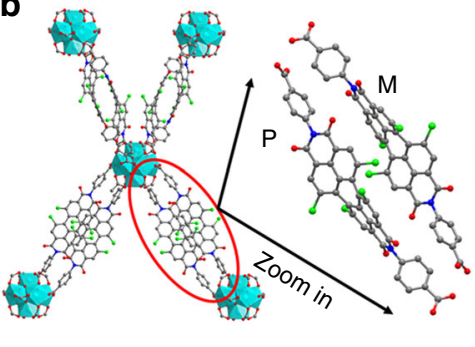

e

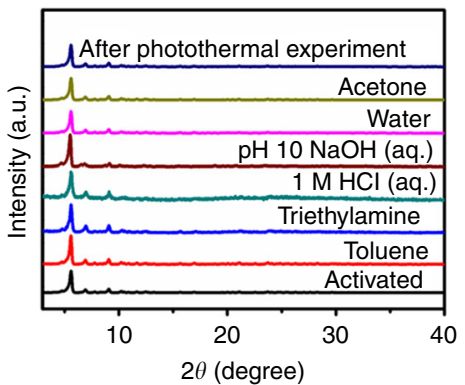

C

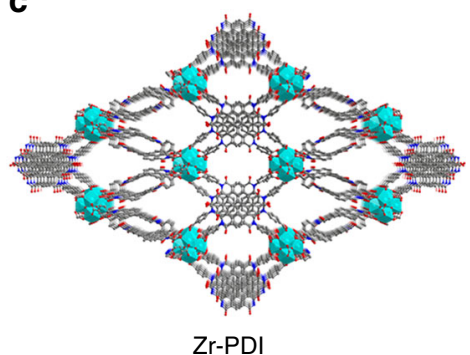

f

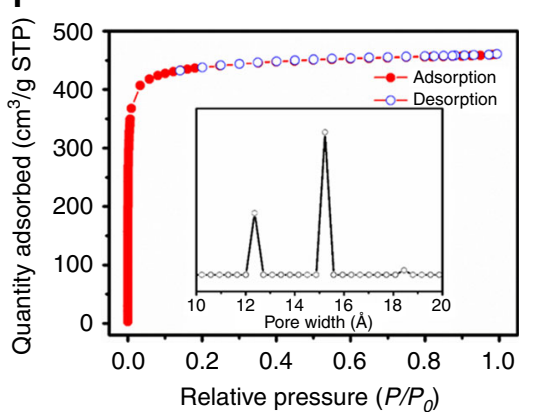

Fig. 1 Synthesis of 3D porous Zr-PDI. a Structures of Zr-cluster and P-2COOH. b Connection mode of Zr-cluster (the Zr-cluster is fully coordinated by 12 carboxylate units) and molecular arrangement of chiral P-2COOH. c a-Axis crystal structure of $\mathrm{Zr}$-PDI. d Single distorted adamantanoid cage (the distorted cage is similar to adamantane) in Zr-PDI. Hydrogen atoms have been omitted for clarity. e Results of stability tests of Zr-PDI incubated under various conditions for $24 \mathrm{~h}$. $\mathrm{Zr}$-PDI powder is washed with acetone and dried under vacuum at $120^{\circ} \mathrm{C}$ prior to measurement. $\mathbf{f} \mathrm{N}_{2}$ sorption isotherm at $77 \mathrm{~K}$ and corresponding pore size distribution

corresponding monomer in DMF, and this shift is due to a combination of excitonic coupling and charge transfer interactions between the $\pi$-stacked PDI chromophores (Supplementary Fig. 13 $)^{41}$. Upon irradiation with blue light $(455 \mathrm{~nm})$ in the presence of TEA vapor as an electron donor, the red Zr-PDI powder turned black in $10 \mathrm{~min}$. The absorption spectrum of $\mathrm{Zr} \mathrm{PDI}^{\bullet}-$ displays three new peaks at 778, 917, and $1008 \mathrm{~nm}$, which are characteristic peaks of $\mathrm{PDI}^{-}-$produced by $\mathrm{PET}^{9,20}$. The new peaks could be assigned to the $D_{0} \rightarrow$ Dn transition with a complex electronic vibration. In comparison, when treated similarly, the $\mathrm{P}-2 \mathrm{COOH}$ crystalline powder (Supplementary Fig. 14) produced only a low yield of $\mathrm{P}-2 \mathrm{COOH}^{--}$, as presented in its absorption spectrum (Supplementary Fig. 15) ${ }^{13}$.

Fluorescence quenching of $\mathrm{Zr}-\mathrm{PDI}^{\bullet}-$ confirmed the formation of RAs (Fig. 2b). There are two fluorescence peaks of Zr-PDI: one is a monomer emission peak at around $578 \mathrm{~nm}$ and the other is a broad peak at around $657 \mathrm{~nm}$ (Supplementary Figs 16-18). The latter can be regarded as an excimer emission from the perylene groups $^{42}$. All experiments and calculations support the proposed PET process demonstrated in Fig. 2d: the excited state $\mathrm{Zr}-\mathrm{PDI}^{\star}$ is reductively quenched by TEA to afford $\mathrm{Zr}-\mathrm{PDI}^{\bullet-}$ and the radical cation $\mathrm{TEA}^{\bullet+}$.

The facile production of $\mathrm{Zr}-\mathrm{PDI}^{\bullet-}$ provides evidence that Zr-PDI has an outstanding ability to adsorb TEA. To calculate the exact sorption capacity of TEA, we measured the TEA sorption test of activated Zr-PDI (MOF without solvent in the pore) sample at $298 \mathrm{~K}$ (Fig. 3a). Zr-PDI gave type I isotherms, with steep uptakes at low absolute pressures. The total TEA uptake capacity at 60 millibars was $4.44 \mathrm{mmol} \mathrm{g}^{-1}$ (or $0.45 \mathrm{~g} \mathrm{~g}^{-1}$ ) for Zr-PDI (Supplementary Fig. 19). Considering the size and molecular weight of TEA, Zr-PDI exhibits high amine capacities with an equilibrium uptake of 9.06 molecules of TEA per $\mathrm{P}-2 \mathrm{COOH}$ molecule ${ }^{43}$. The excellent sorption capacity ensures the efficient PET reaction between TEA and Zr-PDI. The TPA sorption was also measured (Supplementary Fig. 20), the sorption isotherm of TPA being similar to that of TEA. The desorption and adsorption curves nearly coincide, indicating that there is no specific interaction between MOF and amines. The incorporation of TEA inside the framework was then probed by ${ }^{1} \mathrm{H}$ nuclear magnetic resonance (NMR) spectroscopy. After adsorbing TEA vapor, $\mathrm{Zr}$-PDI was dissolved in $\mathrm{NaOH} / \mathrm{D} 2 \mathrm{O}$ solution, and two additional chemical shifts corresponding to the proton signals of $-\mathrm{CH}_{2}$ and $-\mathrm{CH}_{3}$ of TEA are observed in Fig. 3b. After the TEA-loaded Zr-PDI was irradiated with $455 \mathrm{~nm}$ light, two new peaks belonging to $\mathrm{TEA}^{\bullet+}$ emerged. Besides, only broadened resonances belonging to $\mathrm{PDI}$ after irradiation were detected due to the paramagnetic nature of $\mathrm{Zr}-\mathrm{PDI}^{\bullet-}$.

Electron paramagnetic resonance (EPR) spectroscopy was carried out for Zr-PDI samples before and after PET to support the formation of PDI ${ }^{\bullet-}$. As presented in Fig. 3c, Zr-PDI, after treatment with TEA and irradiation, displays a typical EPR signal, confirming the existence of free radicals. The intense resonance has no resolved hyperfine coupling, which is in accordance with literature data $^{20}$. $\mathrm{Zr}-\mathrm{PDI}^{\bullet-}$ and $\mathrm{P}-2 \mathrm{COOH}^{\bullet-}$ have the same $g$ value of 2.0046 (Supplementary Figs 21 and 22), pointing toward the formation of $\mathrm{PDI}^{\bullet-14}$. It has been reported that the integration of the EPR signal is proportional to the yield of RAs $^{13}$. The integration of the EPR signal was calculated to be 21.7 for $\mathrm{P}-2 \mathrm{COOH}^{\bullet-}$ and 3639.1 for $\mathrm{Zr}$-PDI ${ }^{\bullet-}$. Therefore, the yield of $\mathrm{Zr}-\mathrm{PDI}^{\bullet-}$ was about 168 times that of $\mathrm{P}-2 \mathrm{COOH}^{\bullet-}$, consistent with the above UV-Vis-NIR data.

RAs stable under ambient conditions. Most anionic PDI species are readily reoxidized upon exposure to air ${ }^{16-26}$. To evaluate the stability of $\mathrm{Zr}-\mathrm{PDI}^{\bullet-}$, we kept TEA-loaded $\mathrm{Zr}$-PDI ${ }^{\bullet-}$ under ambient conditions (open air and laboratory light) or at different temperatures. Encouragingly, under ambient conditions, $\mathrm{Zr}-\mathrm{PDI}^{\bullet}-$ remained almost unaffected for over a month (Fig. 2a), while the RAs produced by $\mathrm{P}-2 \mathrm{COOH}$ or $\mathrm{P}-2 \mathrm{COOH} / \mathrm{ZrCl}_{4}$ 


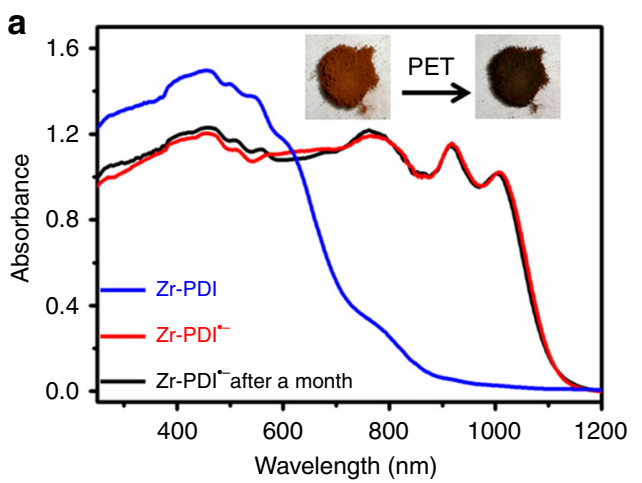

C
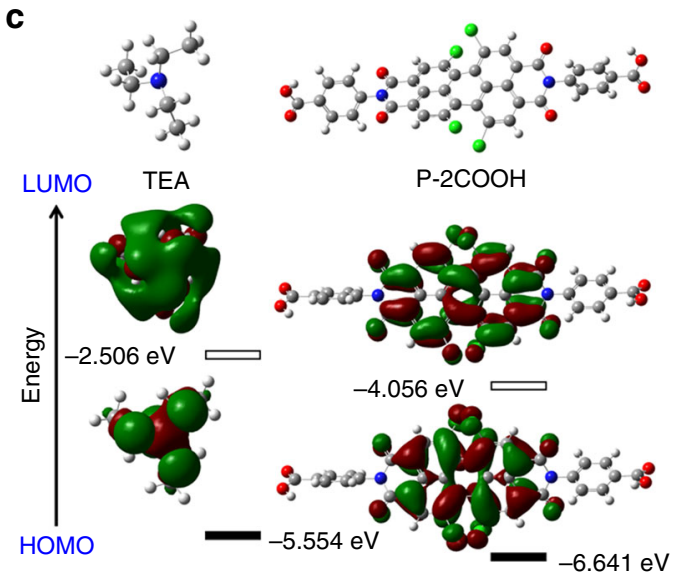

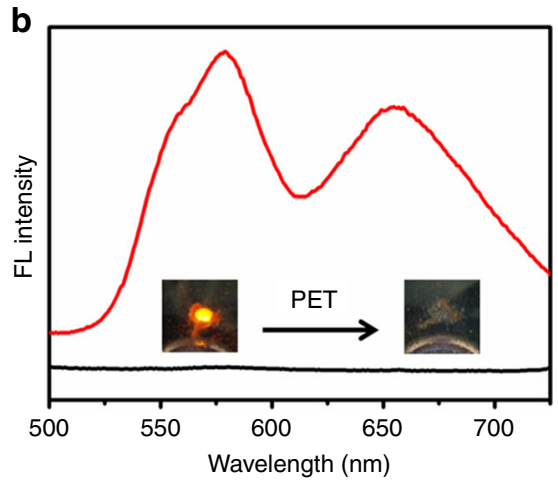

d

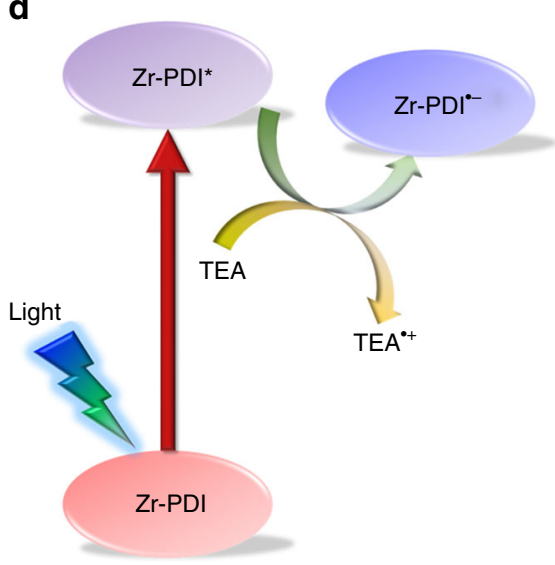

Fig. 2 PET between triethylamine (TEA) and Zr-PDI. a UV-Vis-NIR absorption and $\mathbf{b}$ fluorescence spectra of Zr-PDI before (red line) and after (black line) PET. The insets show the photographs of color and fluorescence changes. $\mathbf{c}$ Energy diagrams optimized by using the B3LYP function and the 6-31G ( $d$ ) basis set and pictorial representations of $\mathrm{HOMO}$ and $\mathrm{LUMO}$ orbitals of TEA and P-2COOH in their ground states. $\mathbf{d}$ Schematic diagram of the PET process between TEA and $\mathrm{Zr}$-PDI, excited state $\mathrm{Zr}-\mathrm{PDI}^{*}$ is reductively quenched by TEA to afford $\mathrm{Zr}$-PDI ${ }^{\bullet-}$

mixtures disappeared completely in $12 \mathrm{~h}$ (Supplementary Fig. 23). Furthermore, $\mathrm{Zr}-\mathrm{PDI}^{\bullet-}$ was rather stable (detected at $778 \mathrm{~nm}$ in UV-Vis-NIR spectra) at high temperatures (retention rates of $89 \%$ at $120^{\circ} \mathrm{C}$ and $76 \%$ even at $180^{\circ} \mathrm{C}$ ) after $1 \mathrm{~h}$ (Supplementary Figs 24 and 25 ), while under the same conditions, P-2COOH${ }^{\bullet-}$ was completely destroyed by oxidation and high temperatures (Supplementary Fig. 26). The porous framework of Zr-PDI appears to stabilize RAs under ambient conditions and at high temperatures. The PET process leading to $\mathrm{Zr}-\mathrm{PDI}{ }^{\bullet-}$ crystalline powder was performed under ambient conditions and required neither protective measures nor low temperatures often necessary for the crystallization of radical ions. Direct format ion of $\mathrm{Zr}-\mathrm{PDI}{ }^{\bullet}-$ by $\mathrm{P}-2 \mathrm{COOH}^{\bullet-}$ was also attempted, but failed to provide $\mathrm{Zr}-\mathrm{PDI}^{\bullet}-$. Obviously, the solvothermal reaction requires high temperature and modulators, so that the RAs will be quenched.

There are three state-of-the-art ambient stable aromatic diimide RAs obtained by covalent modification of aromatic cores ${ }^{17,20,44}$. The displayed stability of $\mathrm{Zr}-\mathrm{PDI}^{\bullet}-$ under ambient conditions is not inferior to that reported in previous studies ${ }^{17,20}$, but without any extra modifications in the tetrachlorinated aromatic core. The present result calls for a closer look on the improved stability of $\mathrm{Zr} \mathrm{PDI}^{\bullet}{ }^{-}$. The PET processes between $\mathrm{Zr}$-PDI and different amines were investigated (Fig. $3 \mathrm{~d}$ ). $\mathrm{Zr}^{-\mathrm{PDI}^{\bullet}-}$ is not supposed to form when large amines $(\sim 15 \AA)$ are used (Supplementary Fig. 27), simply because they cannot enter the pores of $\mathrm{Zr}$-PDI. Some amine-loaded Zr-PDIs ${ }^{\bullet-}$ are presented in Fig. 3f. DEA, EDA, and TEA have comparable sizes of $6.8,5.4$, and $6.6 \AA$ (Supplementary Fig. 28), respectively, which are much smaller than the pore sizes of Zr-PDI ( $\sim 12$ and $\sim 15 \AA)$. Nevertheless, the three amine-loaded $\mathrm{Zr}^{-P D I s}{ }^{-}-$display large differences in stability (Supplementary Fig. 29), so the size of the amine used is irrelevant to the stability of RAs. After TEA-loaded $\mathrm{Zr}$-PDI ${ }^{\circ-}$ were heated for $120^{\circ} \mathrm{C}$, some TEA leaked from $\mathrm{Zr}-\mathrm{PDI}^{\bullet-}$. But, the amount of $\mathrm{TEA}^{\bullet}+$ was constant, and the stability of the residual $\mathrm{Zr}-\mathrm{PDI}^{\bullet-}$ remained almost unchanged for another month under ambient conditions (Supplementary Figs 30 and 31). Thus, the loading of the amine used is irrelevant to the stability of RAs. By comparing amines with different boiling points, we found that the higher the boiling point of the amine used the higher the stability of the $\mathrm{Zr}$-PDI ${ }^{\bullet-}$, as further confirmed by ${ }^{1} \mathrm{H}$ NMR (Supplementary Table 2 and Supplementary Figs 32 and 34). Amines with low boiling points have the tendency to leak from $\mathrm{Zr}^{-\mathrm{PDI}^{-}}{ }^{-}$under ambient conditions, resulting in the instability of RAs. However, amines with high boiling points enable to stay in Zr-PDI under ambient conditions and even at high temperatures. The interpenetrating nature of Zr-PDI leads to a highly porous network with small cages $^{43}$, so it is most likely that high boiling point amines together with amine radical cations are trapped in these cages to form stable adducts. Also, the reduced $\pi-\pi$ stacking of PDIs in the framework prevents RAs to dimerize and quench ${ }^{13}$. Therefore, one can not only improve the yield of RAs but achieve a pronounced stabilization by confining them in the MOF cages.

Photothermal conversion of $\mathrm{Zr}-\mathrm{PDI}^{\bullet-}$. Zr-PDI ${ }^{\bullet-}$ with NIR absorbance can be produced facilely and has extraordinary stability. To further verify and use this stability, we utilized 
a
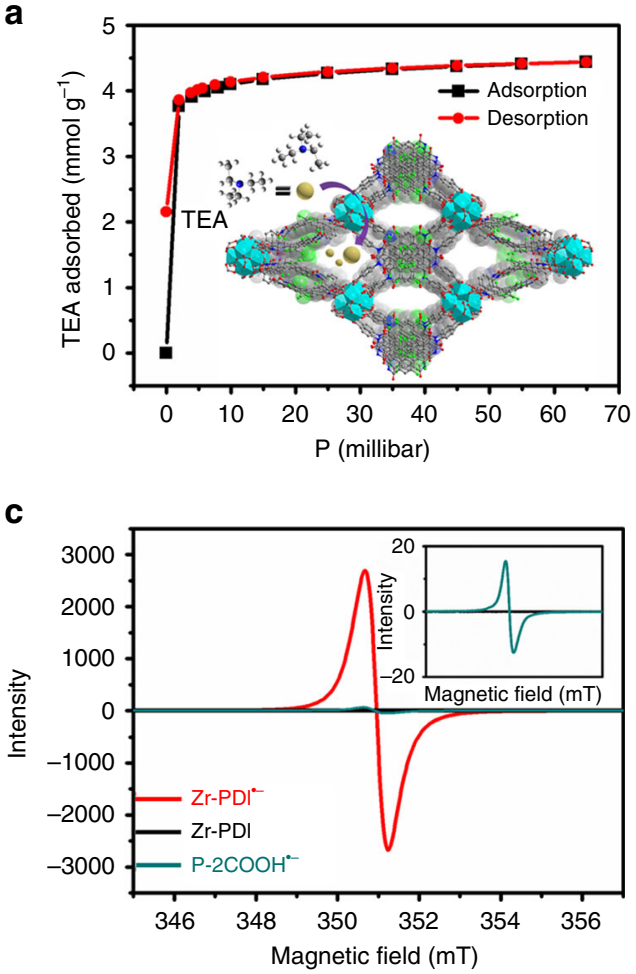

b

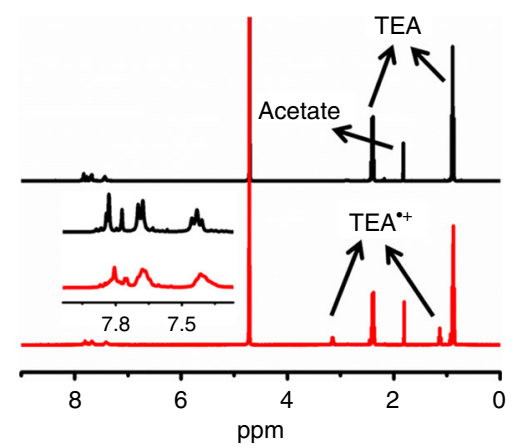

d

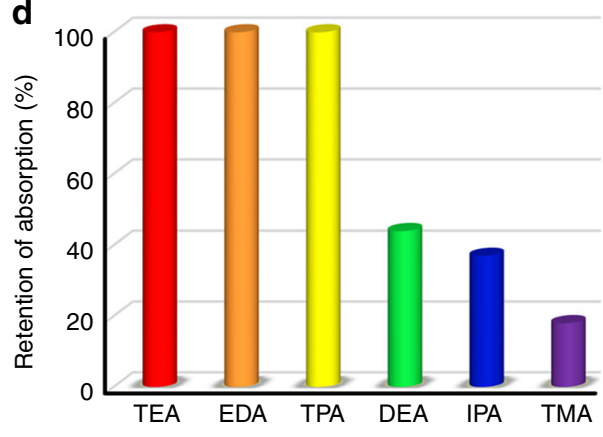

Fig. 3 Characterizations of $\mathrm{Zr}-\mathrm{PDI}{ }^{\bullet-}$ - a TEA adsorption and desorption of activated $\mathrm{Zr}$-PDI; inset shows TEA vapor in $\mathrm{Zr}$-PDI channel. $\mathbf{b}{ }^{1} \mathrm{H}$ NMR spectra of TEA-loaded Zr-PDI before (black line) and after (red line) irradiation; inset shows the peaks of PDI. c EPR spectra of solid $\mathrm{Zr}-\mathrm{PDI}^{{ }^{-}-}$and P-2COOH${ }^{--}$; inset shows the magnified spectrum of $\mathrm{P}-2 \mathrm{COOH}^{*}$. d Stability of $\mathrm{Zr}$-PDI ${ }^{-}-$loaded with different amines under ambient conditions after a week. The ordinate represents the retention rates of the absorption peak at $1008 \mathrm{~nm}$. TEA triethylamine, EDA ethylenediamine, TPA tripropylamine, DEA diethylamine, IPA isopropylamine, TMA trimethylamine

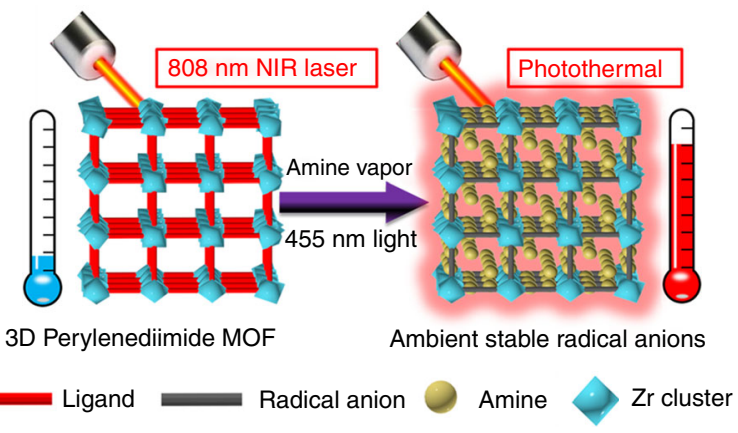

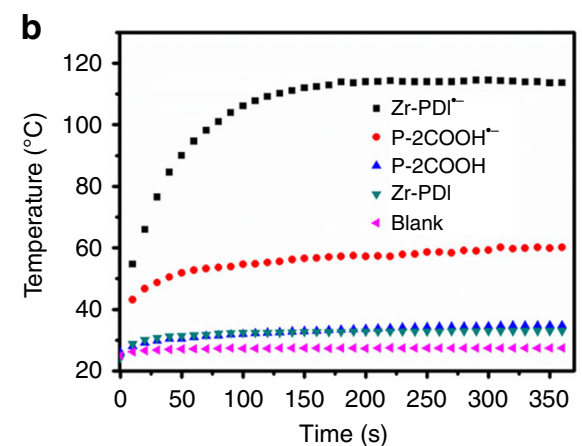
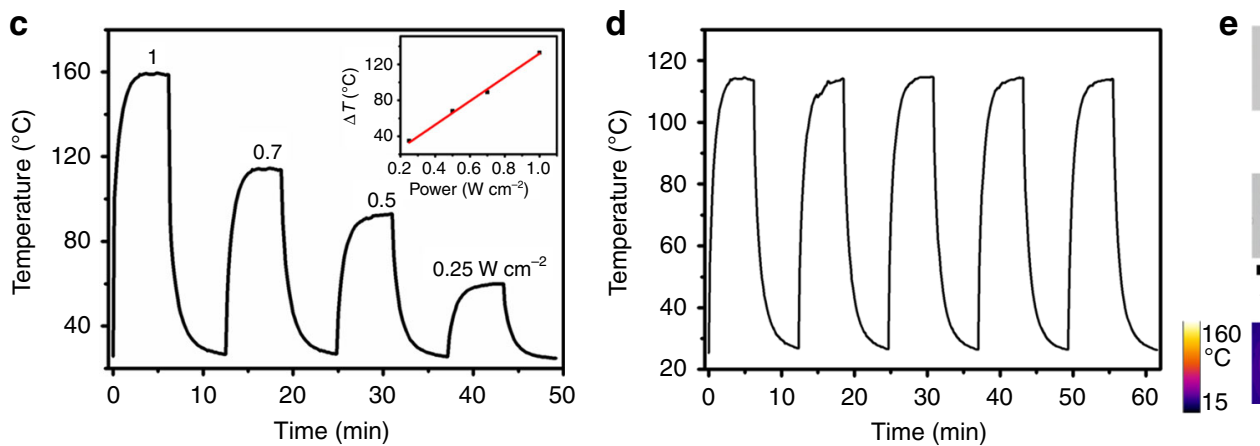

$\mathbf{e}$

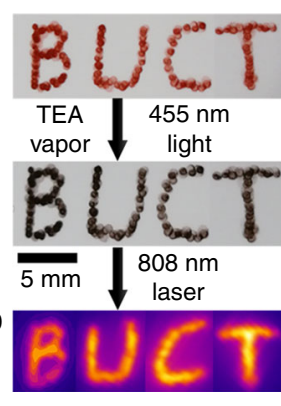

Fig. 4 Photothermal conversion performance of $\mathrm{Zr}-\mathrm{PDI}^{\bullet^{-}}$. a lllustration of the colored $\mathrm{PDI} \mathrm{I}^{\bullet-}$ formation and photothermal conversion of $\mathrm{Zr}-\mathrm{PDI} \mathrm{P}^{\bullet-}$. b Photothermal conversion curves of $\mathrm{Zr}$-PDI ${ }^{--}$film on quartz glass under laser irradiation $\left(808 \mathrm{~nm}, 0.7 \mathrm{~W} \mathrm{~cm}^{-2}\right.$ ). c Temperature rises of $\mathrm{Zr}$-PDI ${ }^{\bullet-}$ at

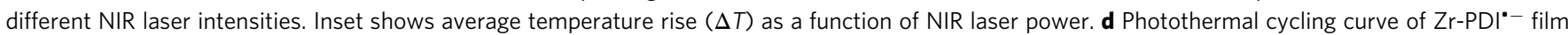
$\left(808 \mathrm{~nm}, 0.7 \mathrm{~W} \mathrm{~cm}^{-2}\right)$. e IR camera images of Zr-PDI-patterned letters BUCT $\left(808 \mathrm{~nm}, 0.7 \mathrm{~W} \mathrm{~cm}^{-2}\right.$ ) 
Zr-PDI ${ }^{\bullet-}$ as a NIR photothermal material (Fig. 4a). Under irradiation with NIR laser $\left(808 \mathrm{~nm}, 0.7 \mathrm{~W} \mathrm{~cm}^{-2}\right)$, the temperature of $\mathrm{Zr}-\mathrm{PDI}^{\circ}-$ powder reached $160^{\circ} \mathrm{C}$ in $10 \mathrm{~s}$ (Supplementary Movie 1). Then to accurately measure the photothermal conversion, $\mathrm{Zr}-\mathrm{PDI}^{\bullet-}$ powder was fixed on a piece of quartz glass to form a uniform film. Under irradiation with $808 \mathrm{~nm}$ laser light $\left.(0.7 \mathrm{~W} \mathrm{~cm})^{2}\right)$, the temperature of the quartz glass was sharply increased to as high as $114^{\circ} \mathrm{C}$, a temperature rise of over $89^{\circ} \mathrm{C}$ within $200 \mathrm{~s}$ (Supplementary Movie 2). In comparison, with NIR laser irradiation, the temperature of a piece of blank quartz glass rose only $2.4^{\circ} \mathrm{C}$, and the temperature of a piece of quartz glass coated with a film of Zr-PDI rose only $9^{\circ} \mathrm{C}$ due to the lack of NIR absorbance (Fig. 4b). These results imply that Zr-PDI - has fascinating photothermal conversion properties, which is comparable to recently reported photothermal conversions of MOFs using UV-Vis light ${ }^{45}$. Lower temperature increases were recorded for $\mathrm{P}-2 \mathrm{COOH}^{\bullet-}$ because of the low yield of RAs, indicating that it cannot serve as a photothermal conversion material. The cooling curve of $\mathrm{Zr}$-PDI ${ }^{\bullet-}$ film, from which the conversion efficiency can be calculated (details are shown in Supplementary Note 1), is presented in Supplementary Fig. 35. An exceptionally high conversion efficiency $(52.3 \%)$ was obtained, much higher than most reported photothermal materials such as Au nanorods (21.0\%), organic cocrystals (18.8\%), and selenophene-derived polymer films (40\%) (Supplementary Table 3) ${ }^{46,47}$. Fig. 4c demonstrates that the photothermal effect is linearly dependent on the NIR laser power from 0.25 to $1 \mathrm{~W} \mathrm{~cm}^{-2}$, an indication of a thermal control performance. Correspondingly, there is no significant light emission in $\mathrm{Zr}^{-\mathrm{PDI}^{\bullet}-}$ (Supplementary Fig. 36), demonstrating the dominant non-radiative transition, in agreement with the high photothermal conversion. Notably, the PXRD pattern of Zr-PDI after laser irradiation is in agreement with the powder data (Fig. 2e), indicating that the crystal structure of Zr-PDI remains intact upon laser irradiation. Zr-PDI after photothermal conversion has a slight decrease in the $\mathrm{N}_{2}$ adsorption, because of the residual $\mathrm{TEA}^{\bullet+}$ in the framework (Supplementary Fig. 37). The cycling test results (Fig. 4c) also demonstrate the high stability of $\mathrm{Zr}$-PDI ${ }^{\bullet}-$ at high temperatures, which was attributed to the effective NIR absorbance of TEA, TPA, EDA-loaded Zr$\mathrm{PDI}^{\bullet}-$ (Supplementary Figs 24 and 25). However, P-2COOH${ }^{\bullet-}$ was unstable at high temperatures and showed inferior cycle test results (Supplementary Fig. 38). The high photothermal conversion of $\mathrm{Zr}-\mathrm{PDI}^{\bullet}-$ convincingly displays the potential of the RAs in photothermal imaging, an illustration of which is shown in Fig. 4e. Under irradiation with NIR light, photothermal materials reveal a significant temperature increase, which can be captured with an IR thermal camera. We then fabricated the letter pattern BUCT with $\mathrm{Zr}-\mathrm{PDI}^{\bullet}-$ powder. Under irradiation with NIR light $\left(808 \mathrm{~nm}, 0.7 \mathrm{~W} \mathrm{~cm}^{-2}\right)$, the powder pattern became imageable with a high resolution. The temperature increase of $\mathrm{Zr}-\mathrm{PDI}^{-}{ }^{-}$is large enough in bio-imaging and biomedical applications such as photothermal therapy ${ }^{48-51}$. Also, the PET process under formation of $\mathrm{Zr}-\mathrm{PDI}^{\bullet-}$ can be initiated by medically active dopamine (Supplementary Figs 39 and 40). To the best of our knowledge, this is the first direct NIR photothermal conversion of a MOF without extra chemical modification by using RAs ${ }^{52-54}$.

In summary, a PDI-based 3D MOF (Zr-PDI) with ultrastable RAs provides a unique platform for NIR photothermal conversion. The suitable pores allow electron donor amine vapors to occupy the cages of Zr-PDI. Upon irradiation with blue light $(455 \mathrm{~nm})$, black $\mathrm{Zr}-\mathrm{PDI}^{\bullet-}$ with NIR absorbance can be formed through PET. The produced RAs, which are in the Zr-PDI cages, can stay unobstructed under ambient conditions for at least a month. So a strategy to stabilize PDI ${ }^{\bullet-}$ without complicated design and tedious synthesis was discovered. Under $808 \mathrm{~nm}$ laser irradiation, the temperature of the $\mathrm{Zr}^{-\mathrm{PDI}^{\bullet}-}$ sharply increases, which has a superior photothermal conversion efficiency of $52.3 \%$ due to non-radiative pathway. With post-synthesis modifications, this MOF material, with outstanding stability, have great potential in biomedical applications such as bio-imaging and photothermal therapy, and the excited RAs may be useful as photoelectrochemical catalysts ${ }^{5,35}$. This work not only provides an efficient method towards ambient stable RAs, but also gives insights into the development of novel PDI materials.

\section{Methods}

General procedure for Zr-PDI preparation. A mixture of $\mathrm{P}-2 \mathrm{COOH}(13.8 \mathrm{mg}$, $0.018 \mathrm{mmol}), \mathrm{ZrCl}_{4}(4.3 \mathrm{mg}, 0.018 \mathrm{mmol})$ was dissolved in $10 \mathrm{~mL} \mathrm{DMF}$ and $0.45 \mathrm{~mL}$ acetic acid. The resulting mixture was heated in a $20 \mathrm{~mL}$ bottle with cap at $90{ }^{\circ} \mathrm{C}$ for 3 days, and then allowed to cool slowly to room temperature. Red crystals were collected and washed with chloroform for three times and dried at vacuum. Yield: $58 \%$ (based on P-2COOH). Anal. Calcd (\%) for $\mathrm{Zr}-\mathrm{PDI}\left(\mathrm{C}_{80} \mathrm{H}_{36} \mathrm{C}_{18} \mathrm{~N}_{4} \mathrm{O}_{27} \mathrm{Zr}_{3}\right)$ : C, 47.01; H, 1.8; N, 2.74. Found: C, 48.53; H, 2.28; N, 2.69 .

Gas adsorption isotherms of Zr-PDI. Nitrogen adsorption isotherms were measured by a volumetric method using a Micromeritics ASAP 2460 gas sorption analyzer. A sample of ca. $111.6 \mathrm{mg} \mathrm{Zr-PDI}$ pre-activated at $100{ }^{\circ} \mathrm{C}$ to remove all residual solvent. Nitrogen isotherms were measured using UHP grade nitrogen. All nitrogen analyses were performed using a liquid nitrogen bath at $-195.8^{\circ} \mathrm{C}$. TEA adsorption isotherms were measured by a gravimetric method using an IGA 002 vapor sorption analyzer. A sample of ca. $33.8 \mathrm{mg} \mathrm{Zr-PDI}$, pre-activated at $100^{\circ} \mathrm{C}$ to remove all residual solvent. TEA adsorption analyses were performed using a water bath at $25^{\circ} \mathrm{C}$ and under vapor pressure of TEA.

Photothermal conversion properties measurement. One milligram Zr-PDI powders were dispersed in $0.1 \mathrm{~mL}$ acetone; the mixture was drop-cast carefully on a quartz glass $(0.8 \times 0.8 \mathrm{~cm}, 320 \mathrm{mg})$ to form a uniform film. The film was then treated with TEA vapor and blue light $(455 \mathrm{~nm})$ for $10 \mathrm{~min}$ to produce $\mathrm{Zr}-\mathrm{PDI}{ }^{\bullet}$ The $808 \mathrm{~nm}$ laser beam was irradiated at a power density from 0.25 to $1 \mathrm{~W} \mathrm{~cm}^{-2}$ The temperature was monitored every $10 \mathrm{~s}$ by a Fluke (Ti400) thermal imaging camera. Photothermal conversion efficiency of the $\mathrm{Zr}-\mathrm{PDI}^{{ }^{-}}$- was calculated by recording the change in the temperature of the quartz glass coated with $\mathrm{Zr}$-PDI powders as a function of time under continuous irradiation of a $808 \mathrm{~nm}$ laser $\left(0.7 \mathrm{~W} \mathrm{~cm}^{-2}\right)$ until the glass reached a steady-state temperature.

\section{Data availability}

The X-ray crystallographic coordinates for structures reported in this study have been deposited at the Cambridge Crystallographic Data Centre (CCDC), under deposition numbers 1859441 . These data can be obtained free of charge from The Cambridge Crystallographic Data Centre via www.ccdc.cam.ac.uk/data_request/cif. The authors declare that other data supporting the findings of this study are available from the corresponding author upon reasonable request.

Received: 8 August 2018 Accepted: 7 January 2019

Published online: 15 February 2019

\section{References}

1. Yang, Y. et al. Atomic-level molybdenum oxide nanorings with full-spectrum absorption and photoresponsive properties. Nat. Commun. 8, 1559 (2017).

2. Robinson, J. T. et al. Ultrasmall reduced graphene oxide with high nearinfrared absorbance for photothermal therapy. J. Am. Chem. Soc. 133, 6825-6831 (2011)

3. Yang, J. et al. Convertible organic nanoparticles for near-infrared photothermal ablation of cancer cells. Angew. Chem. Int. Ed. 50, 441-444 (2011).

4. Lyu, Y. et al. Intraparticle molecular orbital engineering of semiconducting polymer nanoparticles as amplified theranostics for in vivo photoacoustic imaging and photothermal therapy. ACS Nano 10, 4472-4481 (2016).

5. Krapels, K., Driggers, R., Vollmerhausen, R. \& Halford, C. Minimum resolvable temperature difference (MRT): procedure improvements and dynamic MRT. Infrared Phys. Technol. 43, 17-31 (2002).

6. Würthner, F. et al. Perylene bisimide dye assemblies as archetype functional supramolecular materials. Chem. Rev. 116, 962-1052 (2016).

7. Chen, S., Slattum, P., Wang, C. \& Zang, L. Self-assembly of perylene imide molecules into 1D nanostructures: methods, morphologies, and applications. Chem. Rev. 115, 11967-11998 (2015). 
8. Struijk, C. W. et al. Liquid crystalline perylene diimides: architecture and charge carrier mobilities. J. Am. Chem. Soc. 122, 11057-11066 (2000).

9. Lee, S. K. et al. Electrochemistry, spectroscopy and electrogenerated chemiluminescence of perylene, terrylene, and quaterrylene diimides in aprotic solution. J. Am. Chem. Soc. 121, 3513-3520 (1999).

10. Ghosh, I., Ghosh, T., Bardagi, J. I. \& Konig, B. Reduction of aryl halides by consecutive visible light-induced electron transfer processes. Science 346, 725-728 (2014).

11. Zhan, X. W. et al. Rylene and related diimides for organic electronics. Adv. Mater. 23, 268-284 (2011).

12. Che, Y. et al. Enhancing one-dimensional charge transport through intermolecular $\pi$-electron delocalization: conductivity improvement for organic nanobelts. J. Am. Chem. Soc. 129, 6354-6355 (2007).

13. Jiao, Y., Liu, K., Wang, G., Wang, Y. \& Zhang, X. Supramolecular free radicals: near-infrared organic materials with enhanced photothermal conversion. Chem. Sci. 6, 3975-3980 (2015).

14. Yang, Y. et al. Supramolecular radical anions triggered by bacteria in situ for selective photothermal therapy. Angew. Chem. Int. Ed. 56, 16239-16242 (2017).

15. Cui, L. et al. Regulating morphologies and near-infrared photothermal conversion of perylene bisimide via sequence-dependent peptide selfassembly. Chem. Commun. 54, 2208-2211 (2018).

16. Kang, R. et al. Tuning the formation of reductive species of perylene-bisimide derivatives in DMF via aggregation matter. Chem. Commun. 53, 10018-10021 (2017).

17. Kumar, S., Ajayakumar, M. R., Hundal, G. \& Mukhopadhyay, P. Extraordinary stability of naphthalenediimide radical ion and its ultraelectron-deficient precursor: strategic role of the phosphonium group. J. Am. Chem. Soc. 136, 12004-12010 (2014).

18. Kumar, S. \& Mukhopadhyay, P. Ambient stable naphthalenediimide radical ions: synthesis by solvent-free, sonication, mechanical grinding or milling protocols. Green Chem. 20, 4620-4628 (2018).

19. Gosztola, D., Niemczyk, M. P., Svec, W., Lukas, A. S. \& Wasielewski, M. R Excited doublet states of electrochemically generated aromatic imide and diimide radical anions. J. Phys. Chem. A 104, 6545-6551 (2000).

20. Schmidt, D., Bialas, D. \& Würthner, F. Ambient stable zwitterionic perylene bisimide-centered radical. Angew. Chem. Int. Ed. 54, 3611-3614 (2015).

21. Marcon, R. O. \& Brochsztain, S. Highly stable 3,4,9,10-perylenediimide radical anions immobilized in robust zirconium phosphonate self-assembled films. Langmuir 23, 11972-11976 (2007).

22. Shirman, E. et al. Stable aromatic dianion in water. J. Phys. Chem. B 112, 8855-8858 (2008).

23. Iron, M. A., Cohen, R. \& Rybtchinski, B. On the unexpected stability of the dianion of perylene diimide in water-a computational study. J. Phys. Chem. A 115, 2047-2056 (2011)

24. Marcon, R. O. \& Brochsztain, S. Aggregation of 3,4,9,10-perylenediimide radical anions and dianions generated by reduction with dithionite in aqueous solutions. J. Phys. Chem. A 113, 1747-1752 (2009).

25. Bullock, J. E. et al. Photophysics and redox properties of rylene imide and diimide dyes alkylated ortho to the imide groups. J. Phys. Chem. B 114 1794-1802 (2010).

26. Roznyatovskiy, V. V., Gardner, D. M., Eaton, S. W. \& Wasielewski, M. R. Radical anions of trifluoromethylated perylene and naphthalene imide and diimide electron acceptors. Org. Lett. 16, 696-699 (2014).

27. Li, H., Eddaoudi, M., O'Keeffe, M. \& Yaghi, O. M. Design and synthesis of an exceptionally stable and highly porous metal-organic framework. Nature 402, 276-279 (1999).

28. Furukawa, H., Cordova, K. E., O’Keeffe, M. \& Yaghi, O. M. The chemistry and applications of metal-organic frameworks. Science 341, 123044 (2013).

29. Cavka, J. H. et al. A new zirconium inorganic building brick forming metal organic frameworks with exceptional stability. J. Am. Chem. Soc. 130, 13850-13851 (2008).

30. Kitao, T. et al. Hybridization of MOFs and polymers. Chem. Soc. Rev. 46, 3108-3133 (2017).

31. Li, J. R., Kuppler, R. J. \& Zhou, H. C. Selective gas adsorption and separation in metal-organic frameworks. Chem. Soc. Rev. 38, 1477-1504 (2009).

32. Meng, X., Gui, B., Yuan, D., Zeller, M. \& Wang, C. Mechanized azobenzenefunctionalized zirconium metal-organic framework for on-command cargo release. Sci. Adv. 2, e1600480 (2016)

33. Tan, L. L. et al. Stimuli-responsive metal-organic frameworks gated by pillar 5 arene supramolecular switches. Chem. Sci. 6, 1640-1644 (2015).

34. Park, J. et al. Reversible alteration of $\mathrm{CO}_{2}$ adsorption upon photochemical or thermal treatment in a metal-organic framework. J. Am. Chem. Soc. 134, 99-102 (2012).
35. Zeng, L. et al. Organized aggregation makes insoluble perylene diimide efficient for the reduction of aryl halides via consecutive visible light-induced electron-transfer processes. J. Am. Chem. Soc. 138, 3958-3961 (2016).

36. Nelson, A. P., Farha, O. K., Mulfort, K. L. \& Hupp, J. T. Supercritical processing as a route to high internal surface areas and permanent microporosity in metal-organic framework materials. J. Am. Chem. Soc. 131 458-460 (2009).

37. Boer, S. A. et al. Liquid-phase enantioselective chromatographic resolution using interpenetrated, homochiral framework materials. Chem. Eur. J. 20, 11308-11312 (2014).

38. Bellitto, C. et al. Nickel(II) 3,4;9,10-perylenediimide bis-phosphonate pentahydrate: a metal-organic ferromagnetic dye. Inorg. Chem. 51, 7332-7339 (2012).

39. Chen, Z., Debije, M. G., Debaerdemaeker, T., Osswald, P. \& Würthner, F. Tetrachloro-substituted perylene bisimide dyes as promising n-type organic semiconductors: studies on structural, electrochemical and charge transport properties. Chemphyschem 5, 137-140 (2004).

40. Hartnett, P. E. et al. Long-lived charge carrier generation in ordered films of a covalent perylenediimide-diketopyrrolopyrrole-perylenediimide molecule. Chem. Sci. 6, 402-411 (2015).

41. Wu, Y. L. et al. G-quadruplex organic frameworks. Nat. Chem. 9, 466-472 (2017).

42. Kaufmann, C. et al. Ultrafast exciton delocalization, localization, and excimer formation dynamics in a highly defined perylene bisimide quadruple $\pi$-stack. J. Am. Chem. Soc. 140, 4253-4258 (2018).

43. Van Humbeck, J. F. et al. Ammonia capture in porous organic polymers densely functionalized with brønsted acid groups. J. Am. Chem. Soc. 136, 2432-2440 (2014).

44. Kumar, Y., Kumar, S., Mandal, K. \& Mukhopadhyay, P. Isolation of tetracyano-naphthalenediimide and its stable planar radical anion. Angew. Chem. Int. Ed. 57, 16318-16322 (2018).

45. Espín, J., Garzón-Tovar, L., Carné-Sánchez, A., Imaz, I. \& Maspoch, D. Photothermal activation of metal-organic frameworks using a UV-Vis light source. ACS Appl. Mater. Interfaces 10, 9555-9562 (2018).

46. Wang, Y. et al. Cocrystals strategy towards materials for near-infrared photothermal conversion and imaging. Angew. Chem. Int. Ed. 57, 3963-3967 (2018).

47. Kim, B., Shin, H., Park, T., Lim, H. \& Kim, E. NIR-sensitive poly(3,4ethylenedioxyselenophene) derivatives for transparent photo-thermo-electric converters. Adv. Mater. 25, 5483-5489 (2013).

48. Zheng, $\mathrm{X}$. et al. Hypoxia-specific ultrasensitive detection of tumours and cancer cells in vivo. Nat. Commun. 6, 5834 (2015).

49. Zhang, S. et al. Terrylenediimide-based intrinsic theranostic nanomedicines with high photothermal conversion efficiency for photoacoustic imagingguided cancer therapy. ACS Nano 11, 3797-3805 (2017).

50. Liu, C. et al. A water-soluble, NIR-absorbing quaterrylenediimide chromophore for photoacoustic imaging and efficient photothermal cancer therapy. Angew. Chem. Int. Ed. https://doi.org/10.1002/anie.201810541 (2018).

51. Zhang, S. B., Li, J. H., Wei, J. \& Yin, M. Z. Perylenediimide chromophore as an efficient photothermal agent for cancer therapy. Sci. Bull. 63, 101-107 (2018).

52. Meng, J., Chen, X., Tian, Y., Li, Z. \& Zheng, Q. Nanoscale metal-organic frameworks decorated with graphene oxide for magnetic resonance imaging guided photothermal therapy. Chem. Eur. J. 23, 17521-17530 (2017).

53. Chen, Y.-Z. et al. Singlet oxygen-engaged selective photo-oxidation over Pt nanocrystals/porphyrinic MOF: the roles of photothermal effect and Pt electronic state. J. Am. Chem. Soc. 139, 2035-2044 (2017).

54. Wang, W. et al. Nanoscale polymer metal-organic framework hybrids for effective photothermal therapy of colon cancers. Adv. Mater. 28, 9320-9325 (2016).

\section{Acknowledgements}

This work was financially supported by the National Natural Science Foundation of China $(21774007,21574009)$, the Fundamental Research Funds for the Central Universities (PT1811), and Beihuazhongri United Fund (PYBZ1822).

\section{Author contribution}

B.L., K.M., and M.Y. conceived the overall project and elucidated the structure. B.L. and P.L. contributed to experimental work. Y.C. and B.W. contributed to analysis of the data and discussion. All authors contributed to the writing of the manuscript. M.Y. directed the research. 


\section{Additional information}

Supplementary Information accompanies this paper at https://doi.org/10.1038/s41467019-08434-4

Competing interests: The authors declare no competing interests.

Reprints and permission information is available online at http://npg.nature.com/ reprintsandpermissions/

Journal peer review information: Nature Communications thanks Inhar Imaz, Pritam Mukhopadhyay, and the other anonymous reviewer for their contribution to the peer review of this work.

Publisher's note: Springer Nature remains neutral with regard to jurisdictional claims in published maps and institutional affiliations. (c) Open Access This article is licensed under a Creative Commons Attribution 4.0 International License, which permits use, sharing, adaptation, distribution and reproduction in any medium or format, as long as you give appropriate credit to the original author(s) and the source, provide a link to the Creative Commons license, and indicate if changes were made. The images or other third party material in this article are included in the article's Creative Commons license, unless indicated otherwise in a credit line to the material. If material is not included in the article's Creative Commons license and your intended use is not permitted by statutory regulation or exceeds the permitted use, you will need to obtain permission directly from the copyright holder. To view a copy of this license, visit http://creativecommons.org/ licenses/by/4.0/.

(C) The Author(s) 2019 